\title{
Tubular Equivalent Regression Rate in Hybrid Rockets with Complex Geometries
}

\author{
By Landon KAMPS ${ }^{1)}$ and Harunori NAGATA ${ }^{2)}$ \\ 1) Department of Mechanical and Space Engineering, Hokkaido University, Sapporo, Japan \\ 2) Faculty of Engineering, Hokkaido University, Sapporo, Japan
}

(Received June 23rd, 2017)

\begin{abstract}
A new performance parameter titled "tubular equivalent regression rate" is introduced to evaluate burning rates in hybrid rockets with geometrically complex solid propellant grains. Tubular equivalent regression rates are calculated for eight previously reported CAMUI-type hybrid rocket firing tests and compared with extrapolations of previously reported empirical correlations for classic, swirl and vortex hybrid rockets. A non-dimensional number titled "CAMUI Number" is introduced to evaluate how CAMUI-like a solid propellant grain is. The CAMUI Number ranges from 0-1: 0 means no CAMUI-type blocks are used, 1 means only CAMUI-type blocks are used. The results show that the tubular equivalent regression rate increases logarithmically with CAMUI Number, and approaches a value of around $3[\mathrm{~mm} / \mathrm{s}]$ for a CAMUI Number of 1 . This increase in tubular equivalent regression rate is shown to correspond to an increase in performance range from a classic (tubular) hybrid rocket at low CAMUI Numbers $(0.1)$ to surpassing a vortex hybrid rocket for high CAMUI Numbers $(>0.7)$. Furthermore, through the block-by-block analysis of tubular equivalent regression rate in a fuel grain with a CAMUI Number of 0.71 , it is shown that maximum burning rates were achieved in blocks under slightly oxidizer rich conditions.
\end{abstract}

Key Words: Performance, Hybrid Rockets, Regression Rate, Reconstruction Technique

\section{Nomenclature}

\begin{tabular}{ccl}
$a$ & $:$ & power regression coefficient \\
$C$ & $:$ & orifice coefficient \\
$D$ & $:$ & outer diameter \\
$d$ & $:$ & (fuel port) inner diameter \\
$G$ & $:$ & mass flux \\
$L$ & $:$ & length \\
$L_{C A M}$ & $:$ & length of CAMUI section \\
$M$ & $:$ & (fuel) mass unused/remaining \\
$m$ & $:$ & (fuel) mass used/consumed \\
$\dot{m}$ & $:$ & (fuel) mass flowrate \\
$n$ & $:$ & power regression exponent \\
$N$ & $:$ & number of fuel blocks \\
$N_{C A M}$ & $:$ & CAMUI number \\
$O / F$ & $:$ & oxidizer to fuel mass ratio \\
$P$ & $:$ & pressure (at the orifice) \\
$r$ & $:$ & (fuel) port inner radius \\
$\dot{r}$ & $:$ & (fuel) regression rate \\
$t$ & $:$ & time \\
$\Delta t$ & $:$ & time step \\
$\rho$ & $:$ & (fuel) density \\
\hline Subscripts & & \\
$e q$ & $:$ & tubular equivalent \\
$j$ & $:$ & counter for block number \\
$f$ & $:$ & counter for summations \\
$o$ & $:$ & initial \\
stoi & $:$ & oxidizer, to distinguish from fuel \\
&
\end{tabular}

\section{Introduction}

In FY16, Hybrid Rocket Motors (HRMs) were selected over Solid Rocket Motors (SRMs) and Liquid bi-propellant Rocket Motors (LRMs) as the primary propulsion system in numerous aerospace development projects around the world. The most notable projects being the NASA-funded Jet Propulsion Laboratory's (JPL) Mars Ascent Vehicle (MAV) and Virgin Galactic's suborbital spaceplane SpaceShipTwo. ${ }^{1)}$ The largest reasons cited for the selection of HRMs in such applications are safety, low cost and throttealbility, ${ }^{2,3)}$ however the storability and durability of solid fuel grains on the Martian surface were also reported to be crucial to the decision making process for the JPL MAV project. ${ }^{4,5)}$ In whichever example used, the selection of HRMs over alternative propulsion systems in recent development projects is a testament to eight decades of dedicated research and development at universities and industrial laboratories around the world.

Aside from the recent emergence of new HRM development projects, the fact remains that SRMs and LRMs continue to dominate the market. The largest reason cited to explain the selection of SRMs and LRMs over HRMs in practical applications is a poor volumetric loading resulting from a low solid propellant regression rate. ${ }^{6}$ Even though the vacuum specific impulse of HRM propellant combinations is demonstrably larger than that of SRMs, and density-specific impulse is larger than that of LRMs, their low burning rates lead to an in inefficient use of vehicle storage space. In order to ensure adequate mixing and high combustion efficiency, a postcombustion chamber is often added downstream of the primary solid propellant grain, worsening the volumetric load even further. 
The introduction of innovative fuel grain designs to improve the solid propellant burning rates of HRMs has been crucial to achieving powerful and efficient hybrid propulsion systems that can compete with SRMs and LRMs in performance. Over the past two decades, solid propellant grain designs that enhance heat transfer and mixing through clever geometries and improved liquid propellant injection methods, such as in vortex HRMs and Cascaded Multi-stage Impinging-jet (CAMUI) HRMs, have succeeded in significantly improving solid propellant regression rates. ${ }^{5)}$ However, as these designs deviate from the simple tubular and planar fuel grains used to test burning rate theory, quantifying and comparing relative performances of these new designs has also been limited. To elaborate on this point, every fuel block in a CAMUI-type HRM has three major regressing surfaces, each of which has a separate empirical correlation for regression rate. Without knowledge of the exact geometry of the burning surface areas of each block, as well as the overall motor dimensions, a propulsion system developer has no way of quantifying the advantages/disadvantages of selecting this type of motor over others. The same can be said for other complex HRM configurations which have multiple distinct burning surfaces.

The purpose of this paper is to introduce a new performance parameter, titled "tubular equivalent regression rate," that allows for a simplified analysis of burning rates of HRMs of various solid propellant grain designs based on reconstructed fuel consumption histories derived from static firing test data. For the first time, the fuel consumption histories of motors employing CAMUI-type solid fuel grains will be directly compared with those previously reported for tubular, swirl, and vortex HRMs. Additionally, the tubular equivalent regression rate of each block will be estimated and correlated to the local oxidizer-to-fuel mass ratio $O / F$ history to assess the combustion progression in the axial direction.

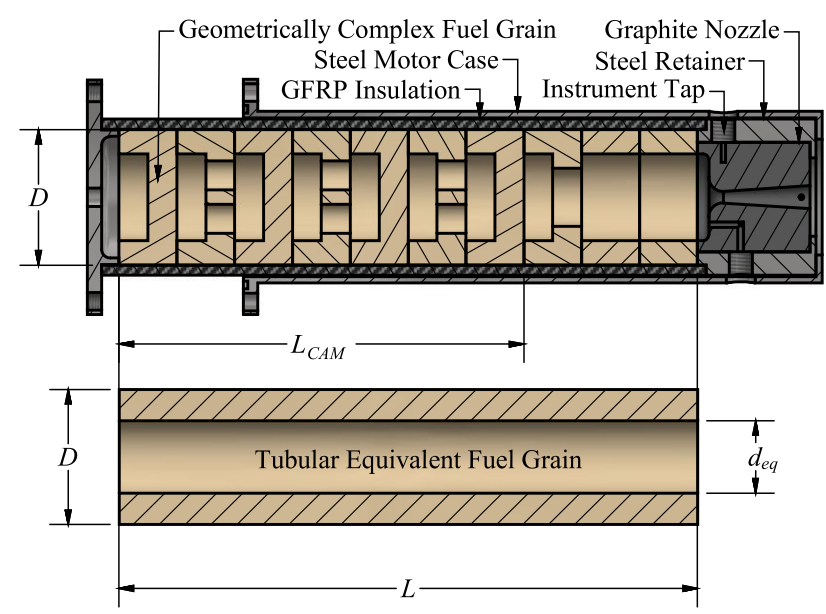

Fig. 1. Cutaway schematic of a generalized CAMUI-type HRM and corresponding tubular equivalent fuel grain.

\section{Method}

The general concept of the tubular equivalent regression rate is to identify the rate of change of the inner radius that a geometrically complex fuel grain would take on by being rearranged into a tube of the same outer dimensions. This is depicted in Fig. 1, in which a CAMUI-type solid fuel grain of length $L$ and outer diameter $D$ corresponds to a tubular equivalent fuel grain of the same outer dimensions but with tubular equivalent inner diameter $d_{e q}$. A fuel grain that combines high fuel filling rates with high fuel consumption rates will be represented by a high tubular equivalent regression rate. For multi-block fuel grains such as those used in CAMUItype HRMs, this concept can be extended to each separate block. Thus, the tubular equivalent radius $r_{e q, i}(t)$ of the $i-t h$ block at any time $t$ can be related to the fuel mass consumption history of that block $m_{i}(t)$ by Eq. (1):

$$
r_{e q, i}(t)=\sqrt{\frac{D^{2}}{4}-\frac{M_{o, i}-m_{i}(t)}{\pi L_{i} \rho}},
$$

where $\rho$ is the density of the fuel, and $L_{i}$ and $M_{o, i}$ are the length and initial fuel mass of the $i$-th block, respectively.

\subsection{Reference tests and fuel configuration}

Fuel mass consumption history $m(t)$ is necessary for determining tubular equivalent radius history $r_{e q}(t)$ according to Eq. (1). The most cost-effective and versatile method for determining $m(t)$ is through the use of reconstruction techniques. The data used in this paper will be adapted from the results in Ref. 7), which were obtained using a new reconstruction technique introduced in that paper as the "Nozzle Throat Reconstruction Technique" or the NTRT. In the NTRT, fuel mass consumption rate history $\dot{m}(t)$ is one of six time-dependent variables calculated as a function of: oxidizer mass flowrate, thrust, chamber pressure, overall fuel mass consumption, and final nozzle throat radius. Numerically integrating the NTRT solution for $\dot{m}(t)$ yields the fuel mass consumption history $m(t)$ necessary for the analysis in this paper.

The results of NTRT for seven previously reported (and one previously unreported) CAMUI-type HRM static firing tests will be referenced for the analysis in the following sections. A summary of the tests used in this analysis is listed in Table 1, and a generalized depiction of the three main types of fuel blocks that make up the fuel grains is shown in Fig. 2.

Table 1. Summary of tests.

\begin{tabular}{ccccc}
\hline Test & Type & Fuel Grain $^{+}$ & Propellants & Reference \\
\hline CAM1 & CAMUI & 1C;1B;1A & O2/HDPE & 7) Test 6 \\
CAM2 & CAMUI & 1C;1B;1A & O2/HDPE & 7) Test 2 \\
CAM3 & CAMUI & 1C;1B;1A & O2/HDPE & 7) Test 5 \\
CAM4 & CAMUI & 2C;1B;1A & O2/HDPE & 7) Test 3 \\
CAM5 & CAMUI & 4C;1B;1A & O2/HDPE & 7) Test 1 \\
CAM6 & CAMUI & 4C;1B;1A & O2/HDPE & 7) Test 4 \\
CAM7 & CAMUI & 9C;1B;1A & O2/HDPE & $10)$ \\
CAM8 & CAMUI & 9C;1B;1A & O2/HDPE & - \\
- & Classic & 1A & O2/HTPB & 6) eq. (64) \\
- & Swirl & 1A & O2/PMMA & 6) Table 5 \\
- & Vortex & 1A & O2/HTPB & 6) eq. (65) \\
\hline
\end{tabular}

+ See Fig. 2 for a depiction of A, B \& C-type fuel blocks; note that the length of fuel blocks varies separately (see Table $2-N_{C A M}$ ) 


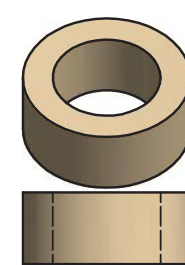

Type A

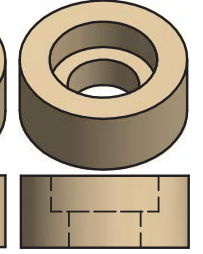

Type B

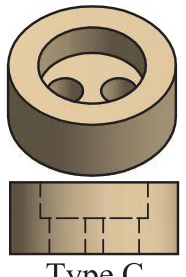

Type C
Fig. 2. The three types of fuel blocks used in CAMUI-type HRMs

Six of the seven previously reported tests, referred to in this paper as CAM1-6, were adopted from Ref. 7), which investigated the relationship between $O / F$ and nozzle erosion rate in $2 \mathrm{kN}$-class CAMUI-type HRMs. The seventh previously reported test, referred to in this paper as CAM7, investigated the nozzle erosion observed in a $15 \mathrm{kN}$-class CAMUI-type HRM. The previously unreported test, referred to in this paper as CAM8, was conducted on a $2 \mathrm{kN}$-class CAMUI-type HRM optimized for flight. Additionally, the regression correlations for three types of tubular HRMs, classic, swirl, and vortex type, will be extrapolated for comparison with the CAMUI-type results.

The benefit of using this selection of data is that the number of CAMUI-type (Type C) fuel blocks used in these experiments varies. This allows us to clarify the improvement in tubular equivalent regression rate based on how CAMUI-like the fuel grain is. However, the specific dimensions of A, B and C-type fuel blocks that were used in the tests referenced for this paper vary in size, and therefore the fuel grain configurations as listed in Table 1 are not enough to quantify the relative length of CAMUI-type sections to non-CAMUI-type sections. For this reason, a new non-dimensional number will be defined, henceforth referred to as the CAMUI Number $N_{C A M}$, which is the ratio of the length of the CAMUI-type fuel section $L_{C A M}$ to length of the fuel grain $L$ :

$$
N_{C A M}=\frac{L_{C A M}}{L} .
$$

A CAMUI Number of 1 means that the entire fuel grain is comprised of C-type blocks, and a CAMUI Number of 0 means that there are no C-type blocks. Note that the difference between $L_{C A M}$ and $L$ is depicted in Fig. 1.

\subsection{Data reduction}

The analysis in the current study is essentially and additional layer of data reduction beyond what has been employed in previous studies. In other words, one of the inputs in the current analysis is an output of a traditional reconstruction technique, in this case the NTRT. Ultimately, the results rely on the same set of static firing test data. As an example, the set of NTRT input data for test CAM8 is plotted in Fig. 3, and the solution for $\dot{m}(t)$ for this test is shown in Fig. 4. It is important to point out that there are non-negligible start-up transients in all of the solutions for $\dot{m}(t)$ referenced for this paper, including test CAM8. Only the times following the designated start point $t_{o}-$ when $\dot{m}(t)$ reaches $75 \%$ of its maximum value - are considered for empirical correlations according to the method introduced in Section 2.3 of this paper. Note that the result of the NTRT for $\dot{m}(t)$ is zero-dimensional, and represents of all the fuel mass that passes through the nozzle.

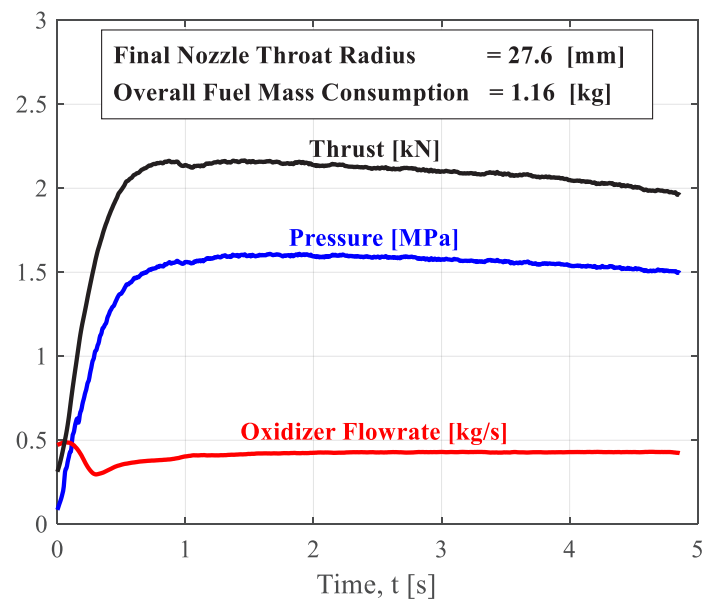

Fig. 3. Experimental inputs for the NTRT in test CAM8.

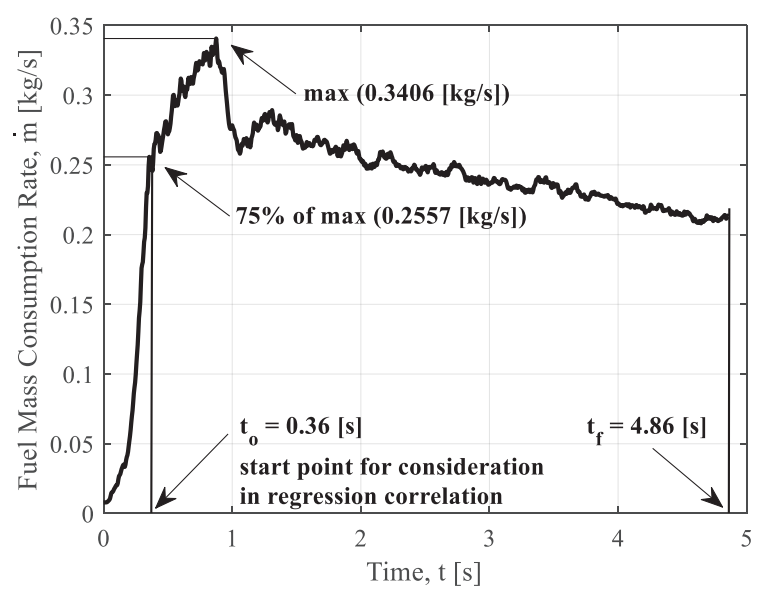

Fig. 4. The NTRT solution for test CAM8.

\subsection{Empirical correlation}

The most common empirical correlation for the solid propellant regression rate $\dot{r}$ in hybrid rockets is Eq. (3):

$$
\begin{aligned}
& \dot{r}=a G_{o x}{ }^{n}, \\
& G_{o x}=\frac{\dot{m}_{o x}}{\pi r^{2}},
\end{aligned}
$$

where $a$ and $n$ are empirically determined constants, and $G_{o x}$ is oxidizer mass flux. Note that $G_{o x}$ is typically expressed in units of $\left[\mathrm{kg} / \mathrm{m}^{2}\right.$-s] whereas $\dot{r}$ is usually expressed in units of $[\mathrm{mm} / \mathrm{s}]$. This empirical correlation is a simplification of the diffusionlimited analysis presented in Ref. 8) that provides a relatively simple and recognizable standard to evaluate performance in tubular HRMs. Accordingly, this study will employ empirical correlation Eq. (3) for the purpose of comparing tubular equivalent regression rate in CAMUI-type HRMs with values previously reported values for other - conventionally tubular HRMs.

When using a power regression to evaluate constants $a$ and $n$ from experimental data, a large number of data points are necessary. This data typically consists of time-averaged values for regression rate and oxidizer mass flux from multiple 
experiments. This method, referred to as end-point method, is cumbersome and expensive. Furthermore, taking spatial and temporal averages of fuel regression rate and oxidizer mass flux often distorts the nonlinear and unsteady behavior of the actual flow conditions during firing. The uncertainty analysis presented by Frederick and Greiner in Ref. 9) highlights the paradigm of balancing benefits of increasing firing duration, which reduces uncertainty due to firing time designation, and of loss in accuracy due to averaging non-linear changes in the cross-sectional area. This problem is exacerbated in CAMUItype hybrid rocket motors due to the coupling of multiple regressing surfaces that follow regression correlations of differing form.

Problems associated with accuracy in end-point calculations for Eq. (3) are avoided in this study by using fuel mass consumption histories obtained by employing NTRT. Whereas in the end-point method dozens of experiments are necessary for an acceptable power regression, using the NTRT allows for a power law regression of the form in Eq. (3) for every single experiment conducted.

\subsection{Algorithm for a singular grain}

When treating a complex fuel grain as the corresponding tubular equivalent, i.e. as one single unit, tubular equivalent regression rate $\dot{r}_{e q}$ is calculated by taking an approximation of the derivative of Eq. (1):

$$
\dot{r}_{e q}(t) \cong \frac{r_{e q}(t+\Delta t)-r_{e q}(t)}{\Delta t},
$$

where $\Delta t$ is the time step resulting from the data sampling rate chosen. Since the tubular equivalent radius can be calculated directly from Eq. (1), the data necessary for carrying out a power regression of the form in Eq. (3) is readily available. The results of Eq. (5) and the associated power regression from test CAM8 are shown in Fig. 5. Due to the large number of data points resulting from the reconstruction technique, the $\mathrm{R}^{2}$ value of the fit is very good $(>95)$. Note that only the data points following $t_{o}$ as defined in Fig. 4 are used in this correlation:



Fig. 5. Power regression in test CAM8.

\subsection{Algorithm for a multi-block grain}

When treating a complex fuel grain as a line-up of several fuel blocks that each have their own tubular equivalent regression rate $\dot{r}_{e q, i}$, the simplified treatment of oxidizer mass flux in Eq. (4) is no longer acceptable. In fact, the original diffusion-limited analysis from which Eq. (3) was derived includes both fuel and oxidizer mass flux in the $G$-term. When using a spatially averaged value for regression rate it is impossible to quantify the position of increased mass flux in the port due to fuel addition, and thus that term is often ignored. However, when considering fuel mass addition by individual fuel blocks, the added mass flux becomes important in employing a power regression of the form in Eq. (3). To avoid confusion, the empirical correlation used in multi-block analysis will be stated separately as Eqs. (6) and (7):

$$
\begin{gathered}
\dot{r}_{i}(t)=a_{i} G_{i}(t)^{n_{i}}, \\
G_{i}(t)=\frac{\dot{m}_{o x}(t)+\dot{m}(t)-\sum_{j=i}^{N} \dot{m}_{j}(t)}{\pi r_{i}(t)^{2}},
\end{gathered}
$$

where $N$ is the total number of blocks that make up the fuel grain, and $G_{i}(t)$ includes mass flux from both the fuel and the oxidizer in that block. Equation (7) allows us to estimate the total mass flux through any block by subtracting the sum of fuel mass flowrates of downstream blocks from the value of fuel mass consumption rate determined by the NTRT. The fuel mass consumption rate in the $i$-th block $m_{i}(t)$ can be represented in terms of the tubular equivalent regression rate of that block:

$$
\dot{m}_{i}(t)=2 \pi \rho L_{i} r_{e q, i}(t) \dot{r}_{e q, i}(t) .
$$

Substituting Eqs. (6) and (7) into Eq. (8) yields an implicit equation for fuel mass flowrate of the $i$-th block $m_{i}(t)$ :

$$
\frac{\dot{m}_{i}(t)}{2 \pi \rho L_{i} r_{e q, i}(t)}=a_{i}\left(\frac{\dot{m}_{o x}(t)+\dot{m}(t)-\sum_{j=i}^{N} \dot{m}_{j}(t)}{\pi r_{e q, i}(t)^{2}}\right)^{n_{i}} .
$$

In order to solve Eq. (9) for $\dot{m}_{i}(t)$ the values of constants $a_{i}$ and $n_{i}$ must be known. As reported in Ref. 6), for boundary layer combustion over a slab of fuel, the exponent $n$ quickly rises in the entrance region of the boundary layer, and settles to a constant value at a non-dimensional axial position of $30 \%$. In other words, in a 10-block tubular fuel grain, the exponents $n_{3}-n_{10}$ can be expected to be approximately the same value. However, due to the irregular geometry of fuel grains referenced for this study, there is no guarantee that the exponents $n_{i}$ can be treated as equal to one another. As a matter of necessity, the values for $n_{i}$ in this study will be set as the value of $n$ that results from the power law regression Eq. (3), i.e. when treating the fuel grain as a singular unit. In this way, only the values of $a_{i}$ are unknown. Since we are assuming that $a_{i}$ are constants, we can iterate for these values based on the overall fuel mass consumption of each block $m_{i}\left(t_{f}\right)$. The overall fuel mass consumption can be approximated by carrying out a simple numerical integration of the form in Eq. (10):

$$
m_{i}(t+\Delta t) \cong \dot{m}_{i}(t) \Delta t+m_{i}(t),
$$

where $m_{i}(t)$ is the fuel mass consumption history resulting from Eq. (9). If the overall fuel mass consumption predicted by Eq. (10) is smaller than the measured value, $a_{i}$ is increased or vice versa. The bi-section iterative technique with a convergence criteria of $10^{-5} \times M_{o, i}$ was used to determine $a_{i}$ in this study. Figure 6 shows the effect of $a_{3}$ on the fuel mass consumption 
history in block 3 of 3 of test CAM1 - the values of $a_{3}$ were taken from the actual iteration history. Lastly, since local fuel consumption is known, it is possible to make an estimate of the local oxidizer to fuel mass ratio $O / F_{i}(t)$ according to Eq. (11):

$$
O / F_{i}(t)=\frac{\dot{m}_{o x}(t)}{\sum_{j=1}^{i} \dot{m}_{j}(t)} .
$$

The sequence of calculations required to solve Eqs. (9) and (11) is depicted in Fig. 7. Calculations start at back end of motor, the position assumed to best represent the solution of the NTRT for fuel mass consumption rate $\dot{m}(t)$. The fuel mass consumption rate of the very last block, block $N$, is found by solving equation Eq. (9), and the correlation coefficient is adjusted to ensure that the integral of the fuel mass consumption history in block $N$ matches the measured value, $m_{N}\left(t_{f}\right)$. This procedure is repeated for the next upstream block and so on, until Eq. (9) has been solved for all blocks. Once Eq. (9) has been solved for all blocks, it is then possible to start at the fore end of the fuel grain and work downstream to calculate the local oxidizer to fuel mass ratio $O / F_{i}(t)$ in reverse order. The

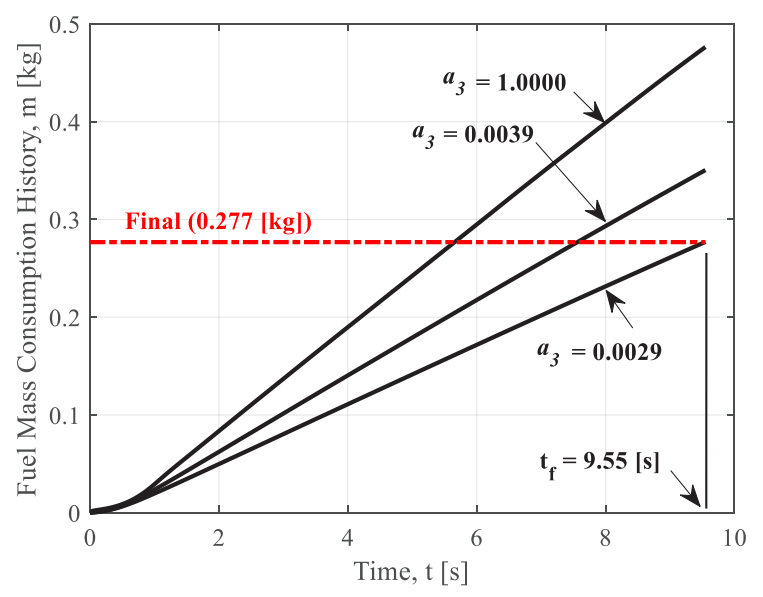

Fig. 6. Effect of $a_{3}$ on fuel consumption in block 3 of 3 in test CAM1.

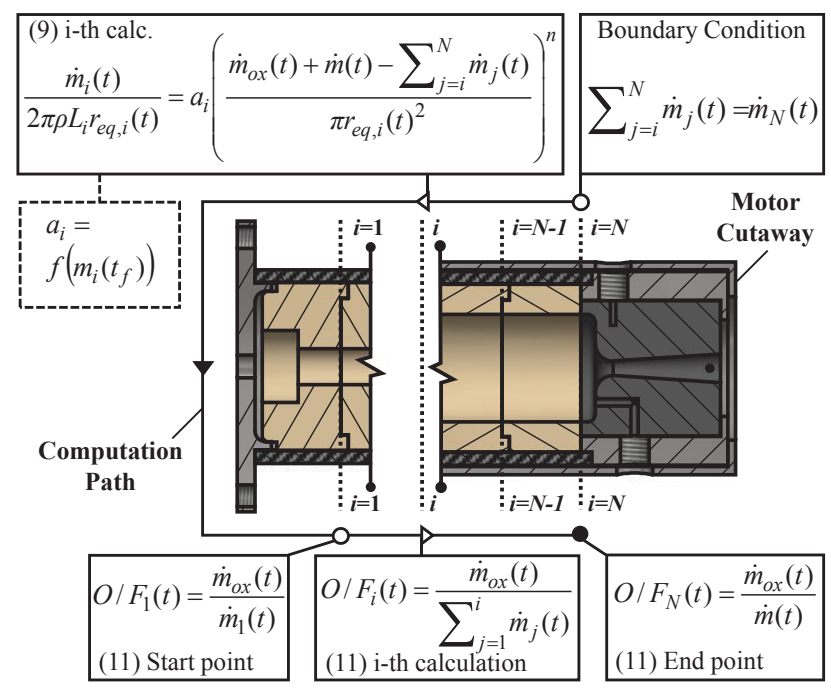

Fig. 7. Flowchart for computations in multi-block analysis. direction of calculations is depicted using arrow heads along the calculation path in Fig. 7, and dotted lines depict the interpretation of the physical location of values solved using this technique.

\subsection{Uncertainty analysis}

The uncertainty in reconstructed solutions is determined by analyzing the uncertainty introduced by experimental measurements. The overall uncertainty $U_{y}$ in some output $y$ is calculated from Eq. (12):

$$
U_{y}^{2}=\sum\left(\frac{\partial y}{\partial x_{j}} U_{x_{j}}\right)^{2},
$$

in which $x_{j}$ represents an input - i.e. one of the experimental measurements $D, L, M_{o, i}, m_{o x}(t), \rho$, or the solution from the NTRT for $\dot{m}(t)$ - and the $U$ terms on the right-hand side represent the uncertainty in those inputs. The uncertainty in fuel mass density $\rho$ was determined experimentally by measuring the mass $M$ and outer dimensions $D$ and $L$ of a cylindrical sample of fuel material prior to manufacturing the grain, and so the uncertainty in this value is derived from the uncertainty in the mass and dimension measurements as follows:

$$
\begin{aligned}
& U_{\rho}^{2}=\sum\left(\frac{\partial \rho}{\partial x_{j}} U_{x_{j}}\right)^{2} \\
& =\left(\frac{\partial \rho}{\partial D} U_{D}\right)^{2}+\left(\frac{\partial \rho}{\partial L} U_{L}\right)^{2}+\left(\frac{\partial \rho}{\partial M} U_{M}\right)^{2} \\
& =\left(\frac{8 M U_{D}}{\pi D^{3} L}\right)^{2}+\left(\frac{4 M U_{L}}{\pi D^{2} L^{2}}\right)^{2}+\left(\frac{4 U_{M}}{\pi D^{2} L}\right)^{2} .
\end{aligned}
$$

The uncertainties in the physical measurements were taken simply to be the precision limits of the sensors used for said measurements. This is because multiple trials with any subject - with the exception of oxidizer mass flowrate which is a timedependent measurement - consistently yielded the same outputs, thus eliminating the need to consider standard deviation in the uncertainty terms. Oxidizer mass flowrate was determined by measuring the pressure drop $\Delta P$ across an orifice plate according to Eq. (14):

$$
\dot{m}_{o x}=C \sqrt{\rho_{o x} \Delta P} .
$$

Here, $C$ is an empirically determined orifice flow coefficient, and $\rho_{o x}$ is the density of liquid oxygen - taken to be $1140 \mathrm{~kg} / \mathrm{m}^{3}$. Two-phase flow was present during the first $0.5 \mathrm{~s}$ of firing in most tests, however the effect of this on the accuracy of $C$ is difficult to quantify, and thus the uncertainty in oxidizer mass flowrate was determined according to equation (15):

$$
\begin{gathered}
U_{\dot{m}_{o x}}^{2}=\sum\left(\frac{\partial \dot{m}_{o x}}{\partial x_{j}} U_{x_{j}}\right)^{2} \\
=\left(\frac{\partial \dot{m}_{o x}}{\partial P_{u p}} U_{P}\right)^{2}+\left(\frac{\partial \dot{m}_{o x}}{\partial P_{d w}} U_{P}\right)^{2},
\end{gathered}
$$

where $P_{u p}$ is the orifice upstream pressure, and $P_{d w}$ is the orifice downstream pressure, such that $\Delta P=P_{u p}-P_{d w}$. The precision of physical measurements taken for this research are summarized in Table 2. 
Table 2. Precision of physical measurements.

\begin{tabular}{llll}
\hline Measurement & Symbol & Apparatus & Precision \\
\hline Diameter & $D$ & MonotaRO digital caliper & $\pm 0.1[\mathrm{~mm}]$ \\
Density & $\rho$ & Eq. (13) & $\pm 6\left[\mathrm{~kg} / \mathrm{m}^{3}\right]$ \\
Flowrate & $\dot{m}_{o x}$ & Eq. (15) & ${ }^{+} \operatorname{var~}[\mathrm{kg} / \mathrm{s}]$ \\
Length & $L$ & MonotaRO digital caliper & $\pm 0.1[\mathrm{~mm}]$ \\
Mass & $M$ & A\&D EK-400i digital scale & $\pm 0.1[\mathrm{~g}]$ \\
Pressure & $P$ & ${ }^{+}$KYOWA PHB-A-10 & $\pm 0.04[\mathrm{MPa}]$ \\
\hline
\end{tabular}

${ }^{+}$this value varies with time, and between tests

\section{Results}

\subsection{Singular grain results}

Figure 8 shows the tubular equivalent regression rate of all tests when the fuel grain is treated as a single unit. Table 3 lists the CAMUI Number and power regression results for $a, n$, and $\mathrm{R}^{2}$ values for all CAMUI-type tests and available data for the power regressions referenced for this paper. The tests CAM1-3 were all conducted with fuel grains that only had one short CAMUI-type (Type C) block at the head-end and were comprised mostly of a long tubular fuel grain. These tests saw tubular equivalent regression rates that fell slightly short of the extrapolation for a classic HRM employing O2/HTPB as propellants. The reason for this is likely explained by the fact that the burning rates for HTPB are notably larger than those of the HDPE used in the CAM-series tests. The test CAM4 had one additional Type C block compared with CAM1-3, and for that reason produced a slightly larger tubular equivalent regression rate. In tests CAM5-6, where four Type $\mathrm{C}$ fuel blocks were used, the tubular equivalent regression rate reaches values in the range of the extrapolation for the swirl HRM. Finally, the two most CAMUI-like fuel grains, CAM7-8, achieved tubular equivalent regression rates in the range of values expected for vortex-type HRMs using an O2/HTPB propellant combination. In all CAM-series tests, the values of $n$ greatly exceeded the theoretical limit of 0.8 for any burning surface that results from Marxman's analysis. ${ }^{8 .)}$ This is most likely the consequence of replacing the larger burning surface area of the complex grains with smaller tubular equivalent burning surface areas.

Figure 9 plots the CAMUI Numbers $N_{C A M}$ against the time averaged tubular equivalent regression rates as listed in Table 3 , revealing a distinctly logarithmic trend. Since the CAMUI Number cannot exceed unity, the maximum expected timeaveraged tubular equivalent regression rate possible with the fuel block design and propellant combination used in these tests appears to be around $3[\mathrm{~mm} / \mathrm{s}]$.

The uncertainties in results as listed in Table 3 and plotted in Fig. 9 are well within acceptable values. The largest relative uncertainties are in the results for correlation coefficient $a$ in tests CAM3-6 at $30 \%-60 \%$. The largest reason this is true is because the values for $a$ are very small, meaning that any uncertainties are relatively large compared with the nominal values. It is also worth noting that these values correspond to the y-intercept of the linear regressions of the histories in Fig. 8. As these values approach zero, the sensitivity to the curve fitting program to changes in input data is likely to be magnified.

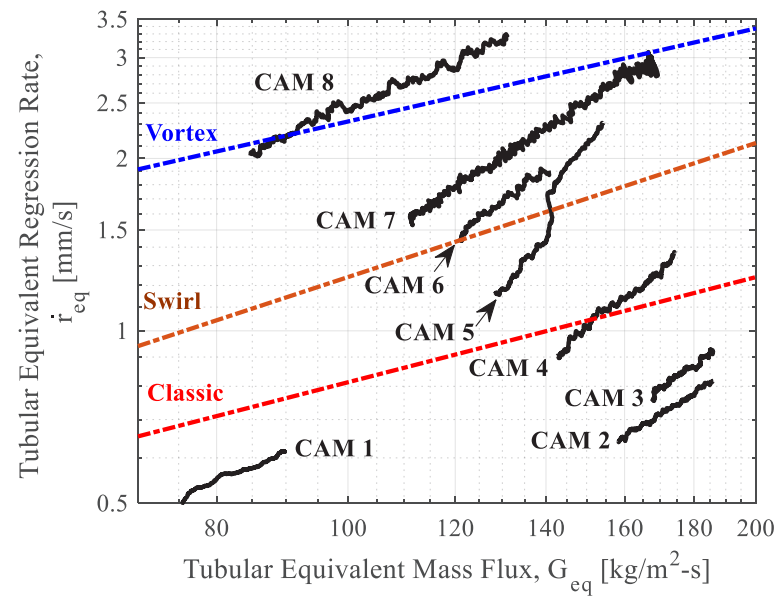

Fig. 8. Comparison of CAMUI-type HRMs with alternative HRMs.

Table 3. Results of singular grain analysis.

\begin{tabular}{cccccc}
\hline Test & $\begin{array}{c}N_{C A M} \\
{[-]}\end{array}$ & $\begin{array}{c}\dot{r}_{e q} \\
{[\mathrm{~mm} / \mathrm{s}]}\end{array}$ & $\begin{array}{c}a \\
{[\mathrm{var}]^{+}}\end{array}$ & $\begin{array}{c}n \\
{[-]}\end{array}$ & $\begin{array}{c}\mathrm{R}^{2} \\
{[-]}\end{array}$ \\
\hline CAM1 & 0.14 & $0.54 \pm 5 \%$ & $0.0058 \pm 15 \%$ & $1.03 \pm 4 \%$ & 0.97 \\
CAM2 & 0.14 & $0.73 \pm 2 \%$ & $3.5 \times 10^{-4} \pm 19 \%$ & $1.49 \pm 3 \%$ & 0.99 \\
CAM3 & 0.14 & $0.83 \pm 2 \%$ & $1.4 \times 10^{-4} \pm 37 \%$ & $1.68 \pm 5 \%$ & 0.97 \\
CAM4 & 0.19 & $1.16 \pm 2 \%$ & $6.4 \times 10^{-5} \pm 30 \%$ & $1.93 \pm 4 \%$ & 0.99 \\
CAM5 & 0.36 & $1.76 \pm 2 \%$ & $1.6 \times 10^{-6} \pm 60 \%$ & $2.82 \pm 6 \%$ & 0.99 \\
CAM6 & 0.36 & $1.83 \pm 3 \%$ & $1.6 \times 10^{-4} \pm 44 \%$ & $1.91 \pm 6 \%$ & 0.97 \\
CAM7 & 0.69 & $2.54 \pm 11 \%$ & $0.0024 \pm 12 \%$ & $1.40 \pm 4 \%$ & 0.95 \\
CAM8 & 0.71 & $2.72 \pm 4 \%$ & $0.0187 \pm 13 \%$ & $1.02 \pm 3 \%$ & 0.99 \\
Classic & 0 & - & 0.0490 & 0.61 & - \\
Swirl & 0 & - & 0.0345 & 0.78 & - \\
Vortex & 0 & - & 0.1930 & 0.54 & - \\
\hline
\end{tabular}

${ }^{+}$Units of $a$ and $n$ depend on one another such that the product of $a \times n$ is in units of $[\mathrm{mm} / \mathrm{s}]$

A breakdown in values for measurement uncertainty in test CAM8 is listed in Table 4. A brief examination of this Table reveals that the uncertainty in the outer diameter $D$ has the greatest influence on the uncertainty in constants $a$ and $n$, whereas the fuel and oxidizer mass flowrates have the greatest influence on the uncertainty in tubular equivalent regression.

Table 4. Breakdown of uncertainty in test CAM8 (singular grain).

\begin{tabular}{cccc}
\hline$\partial x_{i}$ & $\begin{array}{c}\left.\mid \partial \dot{r}_{e q} / \partial x_{i}\right) U_{x_{i}} \mid \\
{[\mathrm{mm} / \mathrm{s}]}\end{array}$ & $\begin{array}{c}\left(\partial a / \partial x_{i}\right) U_{x_{i}} \mid \\
{[\mathrm{var}]^{+}}\end{array}$ & $\begin{array}{c}\left(\partial n / \partial x_{i}\right) U_{x_{i}} \mid \\
{[-]}\end{array}$ \\
\hline $1 \% \dot{m}_{o x}$ & 0.069 & 0.0003 & 0.004 \\
$1 \% m$ & 0.063 & 0.0005 & 0.004 \\
$1 \% D$ & 0.006 & 0.0016 & 0.024 \\
$1 \% L$ & 0.001 & 0.0011 & 0.012 \\
$1 \% M_{o}$ & $<0.001$ & 0.0006 & 0.009 \\
$1 \% \rho$ & 0.005 & 0.0011 & 0.012 \\
\hline
\end{tabular}

\subsection{Multi-block grain results}

When evaluating the fuel flowrate and oxidizer to fuel mass ratio $O / F$ of each block in a multi-block grain according to Eqs. (9) and (11), there appears to be dependency of power regression constants $a_{i}$ on the local oxidizer to fuel mass ratio $O / F_{i}$. This is demonstrated by plotting $a_{i}$ against time-averaged 


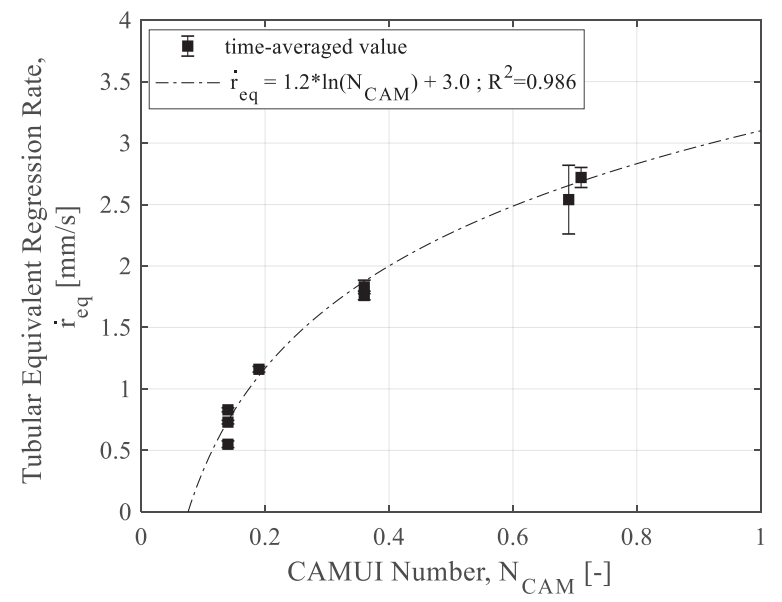

Fig. 9. Increasing tubular equivalent regression rates with increasing CAMUI number in tests CAM1-8.

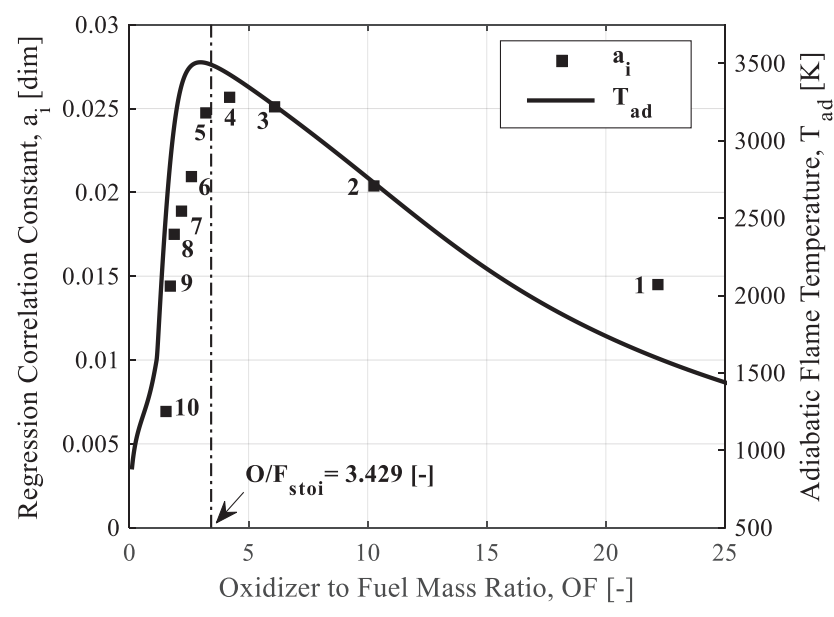

Fig. 10. Regression rates in CAM8 $\left(N_{C A M}=0.71\right)$ blocks are largest where $O / F_{i}$ is near the stoichiometric value.

oxidizer to fuel ratio histories in each block, as shown in Fig. 10. CAM8 test data was chosen to represent this finding because it has the highest CAMUI Number of all the tests included in this study. Based on Fig. 10, the power regression constant appears to reach a maximum value in the $4^{\text {th }}$ block, which has an oxidizer to fuel ratio that is $15 \%$ higher than the stoichiometric value for O2/HDPE of 3.429. This finding differs from the value where the adiabatic flame temperature is largest - 25\% lower than the stoichiometric value. One explanation for the difference in $O / F$ at these maximums may be that the heat transfer to the fuel is not solely dependent on the adiabatic flame temperature. This means that the decrease in adiabatic flame temperature due to an increase in $O / F$ may be counteracted by an increased mass flux at the fuel surface. Another reason for this disagreement may simply be that the assumption that the power regression constants $a_{i}$ are constant is incorrect. In reality, the local $O / F$ in each block is changing with time. This kind of block-specific $O / F$-shift may cause a skew in the trend observed in Fig. 10.

\section{Discussion}

The results as shown in Figs. 8 and 9 clearly make a strong argument for increasing CAMUI Number to improve solid propellant burning rate. Furthermore, CAMUI-type HRMs with CAMUI Numbers above 0.7 can be expected to outperform vortex motors of similar scale and propellant combinations. It is important to point out that HDPE is typically regarded as a slow burning fuel compared with the HTPB fuel used in the vortex motor reference for this comparison. This suggests that the improvement to heat transfer and mixing resulting from the CAMUI fuel grain design allows for the use of stiffer and slower burning fuels without sacrificing a high level of performance. The very large values of $n$ for motors with CAMUI Numbers around 0.4 mean that the tubular equivalent regression rates are particularly sensitive to tubular equivalent mass flux, which is representative of a large shift in $O / F$ during operation. In most cases, this large $O / F$-shift is undesirable, but proved to be useful for investigating the dependency of nozzle throat erosion rate on equivalence ratio in Ref. 7).

Lastly, it is worth noting that the type of analysis carried out in this paper is the first to attempt to directly compare burning rate performance of a geometrically complex solid propellant grain to a tubular one. This kind of analysis can easily be applied to any other hybrid rocket given that the fuel grain outer dimensions are cylindrical. In the case where a tubular solid propellant grain is used, the results of this analysis represent the actual regression rate and not just a tubular equivalent.

\section{Conclusion}

Even though hybrid rockets will play an increasingly important role as a low-cost, safe and versatile chemical propulsion system in future aerospace applications, a way of directly comparing the burning rates of hybrid rocket motors of various innovative and geometrically complex solid propellant designs has yet to be introduced in open literature. A new performance parameter titled "tubular equivalent regression rate" is introduced in this paper to serve this purpose, and stand as a key performance evaluation parameter for all hybrid rockets alongside of specific impulse and/or density specific impulse. Tubular equivalent regression rates were calculated from previously reported CAMUI-type static firing tests data and compared with extrapolations of previously reported regression correlations for classic, swirl and vortex hybrid rockets. Additionally, a new dimensionless parameter titled "CAMUI Number" was introduced to rate how CAMUI-like a multi-stage fuel grain is: 1 for a fuel grain made completely from CAMUI-type blocks, 0 for a fuel grain with no CAMUItype blocks. For CAMUI Numbers around 0.1-0.2, tubular equivalent regression rates were comparable to classic (tubular) hybrid rockets, whereas for high CAMUI Numbers around 0.7, tubular equivalent regression rates were comparable to vortex (tubular) hybrid rockets. This performance parameter can be applied to any type of hybrid rocket motor to allow for a more generalized comparison between new and innovative hybrid rocket designs. 


\section{Acknowledgments}

The test data and results referenced for this paper were made possible because of the hard work and dedication of our senior laboratory members Ryosuke Kawabata, Shota Hirai, and Yuji Saito, and the staff at Uematsu Electric Company. Thank you for your support and guidance throughout this research. This research is supported by the Ministry of Education, Science, Sports and Culture, Grant-in-Aid for Scientific Research (B). 15H04197, 2016.

\section{References}

1) Cecil, O. and Majdalani, J.: Several hybrid rocket technologies hit advanced test stages, Aerospace America, 11 (2016), pp. 54

2) Altman, D. and Holzman, A.: Overview and History of Hybrid Rocket Propulsion, Fundamentals of Hybrid Rocket Combustion and Propulsion, Progress in Astronautics and Aeronautics Vol. 218, Reston, 2007, pp. 1-33.

3) Sutton, G. P. and Biblarz, O.: Hybrid Propellant Rockets, Rocket Propulsion Elements ( $8^{\text {th }}$ ed.), John Wiley \& Sons, New Jersey, 2010, pp. 594-621.

4) Karp, A. C., Nakazono, B., Manrique, J. B., Shotwell, R., Vaughan,
D., and Story, G. T.: A Hybrid Mars Ascent Vehicle Concept for Low Temperature Storage and Operation, AIAA Paper 2016-4962, 2016.

5) Farias, E., Redmond, M., Karp, A. C., Shotwell, R., Mechentel, F. S., and Story, G. T.: Thermal Cycling for Development of Hybrid Fuel for a Notional Mars Ascent Vehicle, AIAA Paper 2016-4563, 2016.

6) Chiaverini, M.: Review of Solid-Fuel Regression Rate Behavior in Classical and Nonclassical Hybrid Rocket Motors, Fundamentals of Hybrid Rocket Combustion and Propulsion, Progress in Astronautics and Aeronautics Vol. 218, Reston, 2007, pp. 37-126.

7) Kamps, L. T., Saito, Y., Kawabata, R.., Wakita, M., Totani, T., Takahashi, Y., and Nagata, H.: Method for Determining Nozzle Throat Erosion History in Hybrid Rockets, J. Propul. Power, 33 (2017), pp. 1369-1377.

8) Marxman, G. A., Wooldridge, C. E., and Muzzy, R. L.: Fundamentals of Hybrid Boundary-layer Combustion, Heterogeneous Combustion, Progress in Astronautics and Aeronautics Vol. 15, 1954, pp. 485-521.

9) Frederick, R. A. and Greiner, B. E.: Laboratory-scale Hybrid Rocket Motor Uncertainty Analysis, J. Propul. Power, 12 (1996), pp. 605611.

10) Saito, Y., Uematsu, T., Isochi, H., Wakita, M., Totani, T., and Nagata, H.: Estimation of Hybrid Rocket Nozzle Throat Erosion History, Trans. JSASS, 14 (2016), pp. 145-151. 